\title{
Correction to: Profiles of Social and Coping Resources in Families of Children with Autism Spectrum Disorder: Relations to Parent and Child Outcomes
}

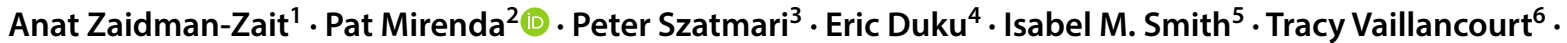 \\ Joanne Volden ${ }^{7}$. Charlotte Waddell ${ }^{8} \cdot$ Teresa Bennett $^{4} \cdot$ Lonnie Zwaigenbaum $^{7} \cdot$ Mayada Elsabaggh $^{9}$. \\ Stelios Georgiades ${ }^{4}$. Wendy J. Ungar ${ }^{10}$. The Pathways in ASD Study Team
}

Published online: 15 February 2018

(c) Springer Science+Business Media, LLC, part of Springer Nature 2018

\section{Correction to: \\ Journal of Autism and Developmental Disorders \\ https://doi.org/10.1007/s10803-018-3467-3}

The original version of this article unfortunately contained a mistake. Wendy J. Ungar was not listed among the authors. The author group has now been corrected.

The original article can be found online at https://doi.org/10.1007/ s10803-018-3467-3.

Anat Zaidman-Zait

anatzaidman@tauex.tau.ac.il

$\triangle$ Pat Mirenda

pat.mirenda@ubc.ca

1 School of Education, Tel Aviv University, P.O. Box 39040, 6997801 Tel Aviv, Israel

2 University of British Columbia, 215 Main Mall, Vancouver, BC V6T 1Z4, Canada

3 Centre for Addiction and Mental Health, University of Toronto, Toronto, ON, Canada

4 McMaster University, Hamilton, ON, Canada

5 Dalhousie University/IWK Health Centre, Halifax, NS, Canada

6 University of Ottawa, Ottawa, ON, Canada

7 University of Alberta, Edmonton, AB, Canada

8 Simon Fraser University, Burnaby, BC, Canada

9 McGill University/Montreal Children's Hospital, Montreal, PQ, Canada

10 University of Toronto, Toronto, ON, Canada 